\title{
Telemonitoring Systems Interoperability Challenge: An Updated Review of the Applicability of ISO/IEEE 11073 Standards for Interoperability in Telemonitoring
}

\author{
M. Galarraga, Student Member, IEEE, L. Serrano, Senior Member, IEEE, I. Martinez, P. de Toledo \\ and Melvin Reynolds, Senior Member, IEEE
}

\begin{abstract}
Advances in Information and Communication Technologies, ICT, are bringing new opportunities and use cases in the field of systems and Personal Health Devices used for the telemonitoring of citizens in Home or Mobile scenarios. At a time of such challenges, this review arises from the need to identify robust technical telemonitoring solutions that are both open and interoperable. These systems demand standardized solutions to be cost effective and to take advantage of standardized operation and interoperability. Thus, the fundamental challenge is to design plug-\&-play devices that, either as individual elements or as components, can be incorporated in a simple way into different Telecare systems, perhaps configuring a personal user network. Moreover, there is an increasing market pressure from companies not traditionally involved in medical markets, asking for a standard for Personal Health Devices, which foresee a vast demand for telemonitoring, wellness, Ambient Assisted Living (AAL) and ehealth applications. However, the newly emerging situations imply very strict requirements for the protocols involved in the communication. The ISO/IEEE 11073 family of standards is adapting and moving in order to face the challenge and might appear the best positioned international standards to reach this goal. This work presents an updated survey of these standards, trying to track the changes that are being fulfilled, and tries to serve as a starting-point for those who want to familiarize themselves with them.
\end{abstract}

\section{INTRODUCTION}

$\mathrm{P}$

atient telemonitoring is one of the most common practices in telemedicine in both indoor and outdoor scenarios, and it is hoped that it can increase the quality of the care and the efficiency of services provided. In fact, it should facilitate a continuous or event monitoring of chronic, elderly, under palliative care or have undergone surgery,

This research work has been partially supported by projects TSI2005-07068-C02-01 and TSI200404940-C02-01 from Ministerio de Educación y Ciencia (Spanish Government), and a personal grant to M.Galarraga from Navarre Regional Government. I.Martínez is with the Communications Technologies Group (GTC), Aragon Institute for Engineering Research (I3A), Univ. Zaragoza (UZ), Spain (e-mail: imr@unizar.es). M.Galarraga, and L.Serrano are with the Electrical and Electronics Engineering Dep., Public Univ. Navarre, Spain (e-mail: miguel.galarraga@unavarra.es). P. de Toledo is with the Bioengineering and Telemedicine Research Centre (GBT) of the Technical Univ. Madrid - Spain. P. de Toledo is now Visiting Professor at the Informatics Dept. Carlos III Univ., Spain. (e-mail: paula@gbt.tfo.upm.es). Melvin Reynolds is with AMS Consulting, Ross-on-Wye, UK (e-mail: MelvinR@AMS-Consulting.co.uk). without them occupying the beds that would be necessary for monitoring in-situ (leaving the beds for the use of patients in a more critical condition). In addition, telemonitored patients can continue to live in their own homes with the subsequent advantages: comfort, more favorable environment, less need for trips to the hospital, etc. Telemonitoring, used appropriately, is expected to decrease healthcare costs.

Two barriers to the current expansion of telemonitoring services, both related to interoperability, can be identified and, in our opinion, they make the transition from pilot experiences to clinical use very difficult: 1) Heterogeneity of devices and systems, and 2) difficulty of integration with healthcare information systems used routinely by healthcare professionals.

For that reason, it is desirable that non-patient oriented devices that form part of a spectrum of use from fitness and wellness monitoring, though devices in support of both independent and assisted living and into self-managed informal monitoring, are also capable of playing a part in such an interoperable continuum of care. As the paradigms for health management change in the face of societal and economic pressures this continuity and flexibility will become increasingly important.

The challenge? In order to be successful in this it will be necessary to follow a globally accepted standard that provides a standardized operation, allows interoperability and provides consistent semantics to recipient systems.

In this paper we provide a starting point and survey of ISO/IEEE 11073 as the best-positioned standard for Plug and Play interoperability of Personal Health Devices. Telemonitoring systems are overviewed in Section II, the option of using ISO/IEEE 11073 standards as the middleware is analyzed in Section III and their evolution is covered in Section IV. Finally, some conclusions are drawn in Section V.

\section{TELEMONITORING SYSTEMS AT A GLANCE}

There have been many different telemonitoring experiments, whether in the home (where the patient measures the necessary parameters and sends the signals to a telemedicine centre), ambulatory - sometimes called ubiquitous or m-health - where the patient uses a mobile device and can therefore undertake monitoring out of the 
home, in controlled environments, such as geriatric residences, or in the framework of consultations with healthcare professionals, etc. Among the most advanced applications are the telemonitoring of diabetic patients [1], heart patients [2-4], respiratory patients [5, 6], and elderly patients [7]. In the majority of cases, the process consists of periodically acquiring vital signals (e.g. blood pressure or heart rate) and other biomedical signals (e.g. ECG signals) to record them locally (home or ambulatory) and later sending them to a remote telemedicine centre, where they are available for the consultation by a specialist or healthcare professional.

The devices used most frequently in telemedicine applications to measure parameters and biological signals are glucose meters, blood pressure and heart rate meters, pulse oximeters, ECG monitors, digital scales, etc. (see Figure 1). The devices can be fixed, but it is increasingly common for them to be wireless or "wearable" (with sensors incorporated into clothing, bracelets, etc.), that makes their use more comfortable. These collections of sensors around the patient make up what can be usually described as either a Body Area Network (BAN) or Personal Area Network (PAN). Often, for monitoring elderly patients or those with limited mobility, these PAN or BAN networks are completed with presence detectors, movement sensors, or similar 'Telecare' devices, which combine to form a Home Area Network (HAN).

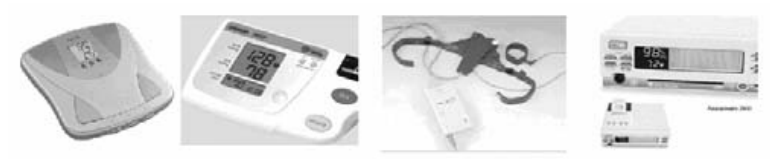

Figure 1 - Typical medical measurement devices

There are two areas of integration that can be identified in the design of a Telemonitoring System that forms part of a Healthcare Information System or a general Telemedicine System:

a. In the local area of the devices, that is the BAN/PAN/HAN network where the patient is located, and where we can find heterogeneous monitoring devices (for example, a sphygmomanometer, scale, or pulse oximeter).

b. In the sphere of Telemedicine Systems, that is in the environment where the patient's medical data has been received, and where, in order to be useful, they must be 1) integrated with the patient healthcare record and 2) accessible by professionals that are taking care of the patient.

The major difficulty in the area of the personal user network is to get different monitoring devices working as a homogeneous network. At present, the manufacturers of telemonitoring devices are using proprietary data exchange formats that are usually not made public. This situation makes it difficult to replace any device (either because they have become obsolete or that better sensors are available, or else because they do not have the necessary usability, do not function correctly, or just because of changes in the needs of the user) when is needed, and also impedes adding new devices to a system without modifying the entire architecture. Any of these situations in one telemonitoring system entails major changes in the application software, not only because of differences in formats, but because the operation paradigm is usually very different. If we consider that is quite common for elements to be replaced, telemonitoring systems need to be designed so they can be integrated in telemedicine platforms in a simple way, as close as possible to the plug and play paradigm. To reach that objective, it is essential to use international standards that can be followed by different manufacturers of devices [8-10]. Avoiding proprietary formats will then decrease the costs in case of replacement, providing high scalability, which is a very important feature in systems that may vary their configuration. The systems can be more centralized and can manage the data captured from the different devices in a more efficient way.

The challenge of having telemonitoring systems that can interoperate and communicate with an open standard is complicated, somehow, because of the features of the devices that are usually implied. Devices and sensors in telemedicine scenarios are usually wearable [9]; these devices need to have some particular electronic features like low voltage-low power, in order to extend the autonomy, limited CPU, reduced size and light weight. Thus, there is a trade-off between the amount of data to be transmitted and these features. The communication protocols need to be lighter, avoiding lengthy communications and being efficient in terms of overhead, bandwidth and use of CPU [9]. With today's means, this leads to the conclusion that the most of intelligence of the systems has to be located away from the MDs or sensors that are monitoring the patient.

For these telemonitoring devices, the transmission technologies may vary, and can be wired or wireless: (e.g. Bluetooth, Zigbee, Wibree, USB, RS-232, etc.). Furthermore, they coexist with other medical devices and network devices such as PCs, routers, modems, mobile phones, etc. that are using different technologies. Then a modular layer design of the standard should have specializations for different low layer communications that can be used.

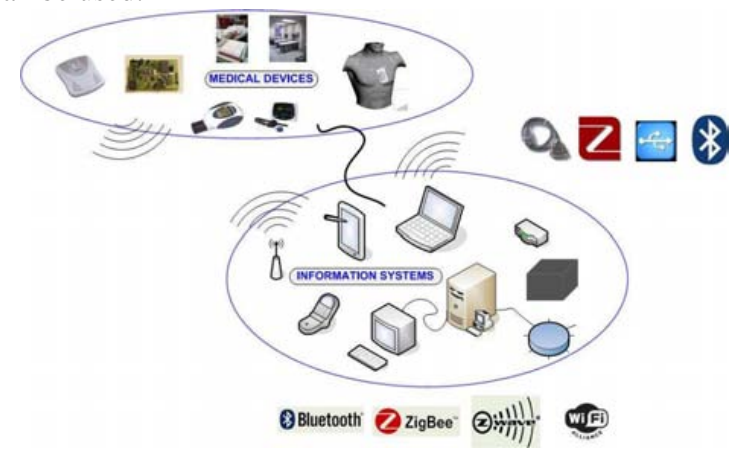

Figure 2 - Medical devices interoperability 
It is also important to mention that a standard for medical device communications in telemonitoring scenarios can change the market and is critical for competitiveness between the different companies, manufacturers and service providers. At this point emerges Continua Alliance, which is a group of technology, healthcare and fitness companies that wish to increase compatibility of e-healthcare devices using the existing standards to create an interoperable framework. Their objectives are to design the guidelines to achieve interoperability of sensors and systems [11].

Currently there is no standard that tackles, specifically, the problem of integrating devices in home and ambulatory telemonitoring environments, but there is a family of standards which purpose is to increase the interoperability of medical devices at the point of care, and that are evolving to include these scenarios. Those are the EN ISO/IEEE 11073 Point-of-Care Medical Device Communication standards [12], which we review here.

To place the standard in context, we summarize other standards in the field of healthcare information systems oriented towards the encoding of signals and biomedical parameters, the standardization of the electronic healthcare record, or the communication between medical applications using standardized messages. Some of these standards are: POCT-1A2 (communication protocols between the device and an access point [12]), Health Level 7 (HL7, for the exchange, management and integration of electronic healthcare information [13]), DICOM-Digital Imaging and Communications in Medicine [14], and EN13606 (for EHR communication [15]).

\section{ISO/IEEE 11073 AS A MIDDLEWARE}

The ISO/IEEE 11073 PoC-MDC (also known as X73) is an internationally harmonized family of standards produced by a grouping of manufacturers, institutions and IEEE in association with ISO and CEN. It consolidates previous IEEE-1073 Medical Information Bus (MIB) [16] and CEN standards (VITAL [17] and INTERMED [18]).

The 11073 standards have been adopted as European standards and will soon be sufficiently complete to replace VITAL and INTERMED which are, formally still valid in Europe.

The European Committee for Standardization (CEN) [19] Technical Committee 251 (TC251) is responsible for health informatics and constitutes the only Europe-wide forum for consensus and standardization of computer science applied to healthcare [20]. It liaises closely with the International Standards Organization (ISO), the principal world standardization body, and for ongoing standardization efforts, the Vienna agreement avoids duplication of items between CEN and ISO.

The 11073 standards address different levels of the ISO OSI reference Model, and have reference models for access to the data, with services and communication protocols for interoperability between medical devices.
In accordance with the 11073 standards, interoperability in the local level of monitoring devices can be solved by connecting all of them with a central element that acts as a main connection integrated compute engine (CE) with the telemonitoring server (see Figure 3). This CE must control the interaction with the different medical devices that form the BAN/PAN network, and monitor the patient (by means of the configuration of the sending and reception of data and control information). In the same way, the $\mathrm{CE}$ will be in charge of connecting the patient network with the telemonitoring server. Of these connections, it is in the communication with the telemonitoring medical devices that compose the patient network where, if widespread use is to be achieved economically, the greatest need for standardization arises, homogenizing the interface between medical devices and the $\mathrm{CE}$.

BAN/PAN/HAN

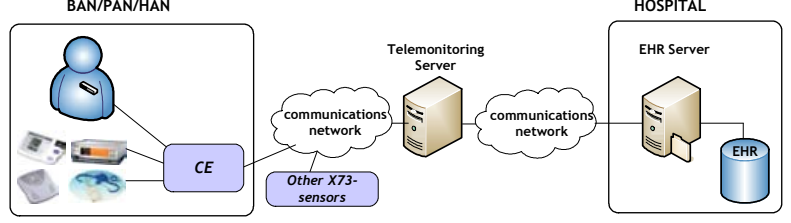

Figure 3-Generic telemedicine integrating heterogeneous systems

In the other critical field of interoperability introduced earlier, integration of a telemedicine system into mainstream healthcare workflow and practice, the main challenge is in being able to incorporate information from perhaps disparate telemonitoring services that themselves include different vendor's medical devices and CEs, managed by the telemonitoring servers; each telemedicine system being connected to the generic Electronic Health Record. In this scenario, middleware technologies provide portability (a telemonitoring system can be connected to different telemedicine systems) and interoperability (medical applications in different clinical environments can exchange information between devices connected to the patient).

A common shortcoming, even when considering use of new technologies, is to overlook the importance of consistent representation of content. This has been a significant problem in the health sector with a number of attempts at achieving consistent representation of meaning having been attempted in the last 20 years or so [21-23]. For medical device communication the problem was recognized as being of major importance when a pan-European project team started work on VITAL [17] - is was simply not possible to correctly interpret between languages the extremely detailed terms being used. The concept of semantic links was adopted to build up language-independent means of describing these detailed concepts. This, allied to a robust information model of the domain [24] facilitated production of a globally usable medical device data language [25] crucial in a global industry fore both devices and health software systems.

The rigorous and extensible nature of the medical device 
data language has been recognized and adopted [26 - 28] to enable large databases to contain physiognomic data for research and regulatory purposes. Work is currently underway to link these detailed representations to the less detailed terms clinicians customarily use - and that are represented in SNOMED CT [22].

It appears likely that only with true semantic interoperability from the device to the health record will it be possible to use operational health information alongside genomic and adverse event databases for data-mining and research to improve practice.

\section{HOW IS ISO/IEEE 11073 FACING THE CHALLENGE?}

IEEE is developing ten telehealth device standards for controlling information exchange to and from personal telehealth devices and cell phones, personal computers, personal health appliances and other computer engines as a part of the ISO/IEEE 11073 family of standards.

The complexity and density of the documents that conform the bulk of the X73 family of standards is one of the key points that are restraining its adoption [29]. To overcome this, the new standards pretend to provide clear definitions of what is needed to implement common communication features for personal telehealth devices, defining also a common core of communication functionality for these devices, and specifying the use of term codes, formats and behaviors in a telehealth environment to favor plug-and-play interoperability.

The new telehealth standards projects are: A technical Report Overview, a Common Networking Infrastructure and an Optimized Exchange Protocol for Medical Device Communication of Personal Health Devices, as well as several Medical Device Specializations. According to IEEE, they will provide the mechanisms needed for real-time, plugand-play interoperability and define comprehensive protocols and services for medical devices in networked operating contexts. [30]. The intention is then to face the challenge and respond evolving with a defined framework, a networking infrastructure and a light communication protocol, appropriated for the kind of Medical Devices, with the special features that we commented in section II, that are found in telemonitoring scenarios.

Due to the different communication technologies that can be used in such a scenario, the ISO/IEEE 11073 family of standards is trying to build a communication standard that is more or less independent of the transport. However, this may be a difficult task as the protocols could be more efficient by using some 'native' features of the communication transport technology that the exchanged information. Even so, the IEEE is making a big effort to solve this trade-off in the most efficient way. In that way, IEEE is building these new standards in collaboration with the Bluetooth SIG (MD-WG) [31], and USB, for example.

According to IEEE, this body of standards will serve a wide range of audiences including medical device and system developers, those who deploy and manage healthcare systems and those who regulate their use, personal telehealth device and compute engine vendors, and institutions that use data from these devices. [30]

The ISO/IEEE 11073 standards are being developed with a high level of international participation and in collaboration with other standards to create interfaces and ensure compatibility between them, as it can be POCT-1A or HL7. In august 2006, the Integrating the Healthcare Enterprise initiative, with the collaboration of ISO/IEEE11073 and HL7 has released the Patient Care Device Technical Framework.

\section{CONCLUSIONS OR FUTURE TRENDS}

Unlike ICU scenarios, the telemonitoring environments involve very strict communications restrictions due to particular electronic features as it was discussed in Section II. The ISO/IEEE 11073 standards are evolving in different ways to face the challenge and provide the basis for an open plug-and-play interoperability for telemonitoring systems.

With appropriate attention to achieving semantic interoperability it may be possible for telemonitoring to take its place alongside acute event monitoring to enable a better understanding of the links between lifestyle, genetics, physiology and pathology so that improvements can be made to the management of health problems.

EN ISO/IEEE 11073 standards appear to be best placed to enable such a continuum to be achieved. Furthermore, the authors' believe is that, at this moment, and with the appropriate changes, $\mathrm{X} 73$ standards can be applicable to telemonitoring scenarios [29,32]. Even though, there is a need for a wider number of platforms that can demonstrate interoperability using these standards, as well as documented implementation examples and IPOSS (Intellectual Property Open Source Software) modules that can be incorporated in such platforms.

\section{REFERENCES}

[1] S. Shea, J. Starren et al., "Project: rationale and design". Columbia University's Informatics for Diabetes Education and Telemedicine (IDEATel). J Am Med Inform Assoc. 2002, 9:49-62.

[2] PA. Ades, FJ. Pashkow et al. "A controlled trial of cardiac rehabilitation in the home setting using electrocardiographic and voice transtelephonic monitoring", Am Heart J. 2000, 139: 543-8.

[3] S. de Lusignan, S. Wells et al., "Compliance and effectiveness of 1 year's home telemonitoring. The report of a pilot study of patients with chronic heart failure", Eur J Heart Fail, 2001, 3: 723-30.

[4] S. Led, L. Serrano, M. Galarraga, "Wearable wireless monitoring system based on bluetooth technology: a tutorial", IFMBE Proceedings EMBEC'05, 3rd European Medical \& Biological Engineering Conference, IFMBE European Conference on Biomedical Engineering, (Vol. 11). IFMBE, 2005.

[5] J. Finkelstein, MR. Cabrera et al., "Internet-based home asthma telemonitoring: can patients handle the technology?", Chest, 2000, 117: 148-55.

[6] B. Morlion, Y. Verbandt et al. "A telemanagement system for home follow-up of respiratory patients", IEEE Eng Med Biol Mag, 1999, 18: 71-9.

[7] B. Johnston, L. Wheeler et al. "Outcomes of the Kaiser Permanente Tele-home health research project”. Arch Fam Med, 2000, 9:40-5. 
[8] S. Warren, J. Yao, R. Schmitz and J. Lebak, "Reconfigurable pointof-care systems designed with interoperability standards," Annual International Conference of the IEEE Engineering in Medicine and Biology - Proceedings, 2004. vol. 26 V, pp. 3270-3273

[9] J. Yao and S. Warren, "Applying the ISO/IEEE 11073 standards to wearable home health monitoring systems," Journal of Clinical Monitoring and Computing, vol. 19, 2005, pp. 427-436

[10] M. Galarraga, L. Serrano, "The Need for Standards in Medical Device Interoperability: Why and Where?', IFMBE Proceedings EMBEC'06, 5th European Medical \& Biological Engineering Conference, IFMBE European Conference on Biomedical Engineering, (vol 12). IFMBE, 2006

[11] Continua Alliance web site: http://www.continuaalliance.org/home/

[12] "Point-of-Care Connectivity; Approved Standard- Second Edition Preview Sample Pages," 2006, http://www.clsi.org/source/orders/

[13] HL7. Health Level Seven, Devices Special Interest Group. http://www.hl7.org/Special/committees/healthcaredevices/index.cfm.

[14] DICOM. http://medical.nema.org/.

[15] IEEE 1073:1996, IEEE Standard for Medical Device Communications - Overview and Framework. IEEE, ISBN: 1-55937755-0.

[16] IEEE 1073:1996, IEEE Standard for Medical Device Communications - Overview and Framework. IEEE, ISBN: 1-55937755-0

[17] ENV 13734:2000, Health informatics - Vital signs information representation (also known as VITAL). CEN, 2000

[18] ENV 13735:2000, Health informatics - Interoperability of patient connected medical devices (also known as INTERMED). CEN, 2000

[19] Comité European de Normalisation (CEN). http://www.cen.eu

[20] Comité European de Normalisation, Technical Committee 251 Health informatics (CEN/TC251). http://www.centc251.org.

[21] NHS Clinical Terms (also known as Read Codes), http://www.connectingforhealth.nhs.uk/terminology/readcodes/earlier versions

[22] SNOMED (formerly Systematized Nomenclature of Medicine), http://www.snomed.org

[23] Huff SM, Rocha RA et al. Development of the Logical Observation Identifier Names and Codes (LOINC) vocabulary J Am Med Inform Assoc. 1998, 5:276-92. See too http://www.regenstrief.org/medinformatics/loinc

[24] EN ISO 11073-10201:2005, Health informatics - Point-of-care medical device communication - Part 10201: Domain information model. CEN, 2005

[25] EN ISO 11073-10101:2005, Health informatics - Point-of-care medical device communication - Part 10101: Nomenclature. CEN, 2005

[26] HL7 Technical Committee for Regulated Clinical Research Information Management (RCRIM), http://www.hl7.org/special/Committees/rcrim/rcrim.htm. Specifically RCRIM - Annotated ECG WG: http://www.hl7.org/special/committees/list_sub.cfm?list=rcrimaecg

[27] HL7 Technical Committee for Orders and Observations, Implantable Devices - Cardiac WG (IDC), http://www.hl7.org/special/Committees/orders/orders.htm

[28] International Organization for Terminology in Anesthesia (IOTA) http://www.apsf.org/initiatives/data_dictionary.mspx

[29] I. Martínez, J. Fernández, M. Galarraga, L. Serrano, P. de Toledo, S. Jiménez-Fernández, S. Led, M. Martínez-Espronceda and J. García, "Implementation of an End-to-End Standards-based Patient Monitoring Solution", IEE Proceedings Communications - Special Issue on Telemedicine and e-Health Communication Systems COM2006-0703, accepted for publication,. 2007

[30] Institute of Electrical and Electronics Engineers Inc. (IEEE).

[31] Bluetooth SIG web site: http://www.bluetooth.com/Bluetooth/SIG/

[32] P. de Toledo, M. Galarraga, I. Martínez, L. Serrano, J. Fernández and F. Del Pozo, "Towards e-Health Device Interoperability: The Spanish Experience in the Telemedicine Research Network" Conference Proceedings, IEEE EMBC, 2006, 28th Annual International Conference of the Engineering in Medicine and Biology Society, 2006. 